\title{
On a Problem About Covering Lines by Squares
}

\author{
Walter Kern and Alfred Wanka \\ Mathematisches Institut der Universität zu Köln, Weyertal 86-90, \\ D-5000 Köln 41, Federal Republic of Germany
}

\begin{abstract}
Let $S$ be the square $[0, n]^{2}$ of side length $n \in \mathbb{N}$ and let $\mathscr{S}=\left\{S_{1}, \ldots, S_{1}\right\}$ be a set of unit squares lying inside $S$, whose sides are parallel to those of $S . \mathscr{S}$ is called a line cover, if every line intersecting $S$ also intersects some $S_{i} \in \mathscr{S}$. Let $\tau(n)$ denote the minimum cardinality of a line cover, and let $\tau^{\prime}(n)$ be defined in the same way, except that we restrict our attention to lines which are parallel to either one of the axes or one of the diagonals of $S$. It has been conjectured by Fejes Tóth that $\tau(n)=2 n+O(1)$ and by Bárány and Füredi that $\tau^{\prime}(n)=\frac{3}{2} n+O(1)$. We will prove that, instead, $\tau^{\prime}(n)=\frac{4}{3} n+O(1)$ and, as to Fejes Tóth's conjecture, we will exhibit a "noninteger" solution to a related LP-relaxation, which has size equal to $\frac{3}{2} n+O(1)$.
\end{abstract}

\section{Introduction}

The following conjecture is due to Fejes Tóth [2], [3]: "Given $n$ points in the unit square, there exists a line intersecting the unit square, which has $L_{\infty}$-distance at least $1 /(n+1)$ from each point."

This can obviously be restated as follows: let $S$ denote the square $[0, n]^{2}$ of side length $n$ and let $\mathscr{S}=\left\{S_{1}, \ldots, S_{t}\right\}$ be any collection of unit squares lying inside $S$. Let us say, that $\mathscr{P}$ is a line cover for $n$ of size $t$, if every line which intersects $S$ also intersects a member of $\mathscr{P}$. (Let us agree that "intersect" shall mean "have a point in common.") Furthermore, let $\tau(n)$ denote the smallest $t \in \mathbb{N}$ such that there exists a line cover for $n$ of size $t$. Then, for $n \in \mathbb{N}$ odd, Fejes Tóth's conjecture states that $\tau(n)=2 n-1$ (see Fig. 1).

If one cannot solve a problem, the most natural thing to do is to consider another one. Thus, in a paper by Bárány and Füredi [1], the function $\tau^{\prime}(n)$ is defined in the very same way as $\tau(n)$, except that we want to cover only those lines which are parallel to one of the sides or diagonals of $S$. For $n \in \mathbb{N}$ odd, they proved that $\tau^{\prime}(n) \geq \frac{4}{3} n-\frac{1}{3}$ and conjectured that $\tau^{\prime}(n)=\frac{3}{2} n+O(1)$ would be the 


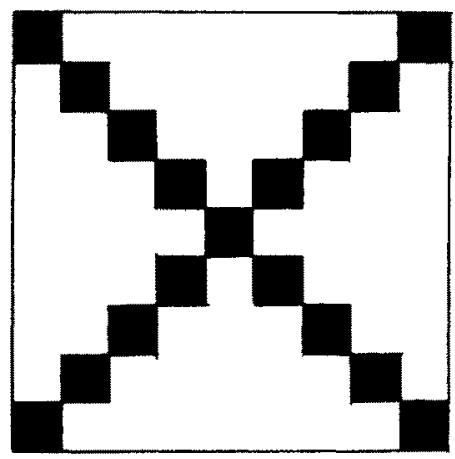

Fig. 1.

correct value. However, as we will see, there do exist coverings of size $\frac{4}{3} n+O(1)$, showing that $\tau^{\prime}(n)=\frac{4}{3} n+O(1)$.

\section{The LP-relaxation}

It seems to be likely, that in an optimal covering $\mathscr{S}=\left\{S_{1}, \ldots, S_{i}\right\}$ the small squares $S_{i}$ may be chosen such that their vertices are in $\mathbb{N}^{2}$. However, we did not succeed in proving this. Nevertheless, we may restrict our attention to coverings of this type. Then the problem readily translates into a standard covering problem, say $(P)$, whose $0-1$ solutions correspond to line covers. To every subsquare $S_{i j}:=$ $[i, i+1] \times[j, j+1], 0 \leq i, j \leq n-1$ we associate a variable $x_{i j}$, and to every line $g$ intersecting $S$, we associate its incidence vector in $\{0,1\}^{n^{2}}$, which has a 1-entry in position $(i, j)$ if $g$ meets $S_{i j}$ (see Fig. 2).

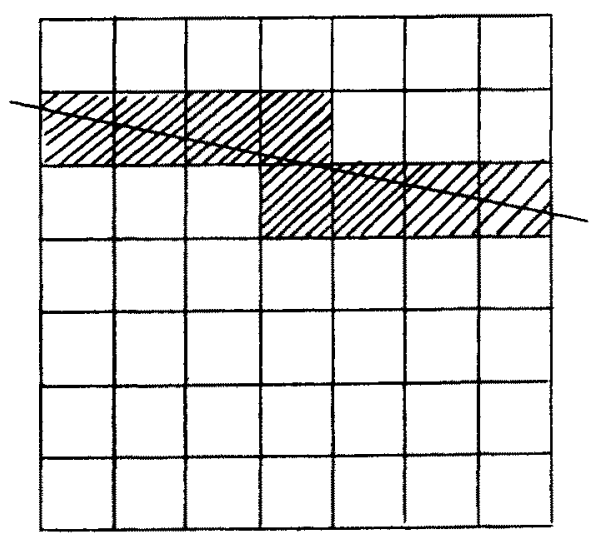

Fig. 2. 
Let $G$ denote the 0-1 matrix, whose rows are the incidence vectors of lines as above. Then our covering problem reads as follows:

$$
\begin{array}{r}
\min 1^{T} x \\
G x \geq 1, \\
x \geq 0 .
\end{array}
$$

Then, as we already noted, every $0-1$ solution $x=\left(x_{i f}\right)$ corresponds to a line cover $\mathscr{S}$.

The dual of $(P)$, which we denote by $\left(P^{*}\right)$, is a "(line-) packing" problem:

$$
\begin{aligned}
& \max 1 u \\
& u^{T} G \leq 1 \\
& u \geq 0
\end{aligned}
$$

Every feasible solution of $\left(P^{*}\right)$ provides us with a lower bound for the size of a line cover $\mathscr{S}$. And, in fact, this is (essentially) the way Bárány and Füredi proved that $\tau^{\prime}(n) \geq \frac{4}{3} n-\frac{1}{3}$. We include their short (and clever) proof for completeness.

For notational convenience we transform the problem as follows: let $Q$ denote the square $[-1,1]^{2}$ and let $\varepsilon:=1 / n$. Thus elements of a line cover become small squares of side length $2 \varepsilon$. Now consider all lines $g$, which intersect $Q$ and are parallel to one of the axes or diagonals of $Q$. Assign weights (dual variables) to these lines in the following way:

If $g$ denotes the line $y=c$ or $x=c$, let $w(g):=\frac{1}{2}-\frac{1}{2} c^{2}$.

If $g$ denotes the line $y=x+h$ or $y=-x+h$, let $w(g):=\frac{1}{8} h^{2}$.

Then a straightforward computation [1] shows that the total weight of the lines is

$$
2 \int_{-1}^{+1}\left(\frac{1}{2}-\frac{1}{2} c^{2}\right) d c+2 \int_{-2}^{+2} \frac{1}{8} h^{2} d h=\frac{8}{3}
$$

Furthermore, the total weight of all lines meeting a small square of side length $2 \varepsilon$ can be shown to be equal to $2 \varepsilon+\frac{2}{3} \varepsilon^{3}$ (independent of the position of the small square inside $Q$ ). From this, one concludes that, if $\mathscr{S}$ is any line cover of size $t$, $t \geq \frac{8}{3} /\left(2 \varepsilon+\frac{2}{3} \varepsilon^{3}\right)>\frac{4}{3} n-\frac{1}{3}$.

On the other hand, there exists a feasible solution $\bar{C}$ for $(P)$, having objective function value $\frac{4}{3}(n+1)$. This is illustrated in Fig. 3. Note that this is indeed feasible, since every line parallel to one of the diagonals meets precisely two adjacent small subsquares $S_{i j}$ at the boundary of $S$.

What can we learn from this? The most striking fact is that our solution for $(P)$ is nonzero only at the boundary of $S$ and on the diagonals. And, in fact, if we look at the weights defined for the dual problem, we can see that every "good" 


\begin{tabular}{|c|c|c|c|c|c|c|}
\hline 1 & $1 / 6$ & $1 / 6$ & $1 / 6$ & $1 / 6$ & $1 / 6$ & 1 \\
\hline $1 / 6$ & $1 / 3$ & & & & $1 / 3$ & $1 / 6$ \\
\hline $1 / 3$ & & $1 / 3$ & & $1 / 3$ & & $1 / 6$ \\
\hline $1 / 6$ & & & $2 / 3$ & & & $1 / 6$ \\
\hline $1 / 6$ & & $1 / 3$ & & $1 / 3$ & & $1 / 6$ \\
\hline $1 / 6$ & $1 / 3$ & & & & $1 / 6$ & $1 / 3$ \\
\hline 1 & $1 / 6$ & $1 / 6$ & $1 / 6$ & $1 / 6$ & $1 / 6$ & 1 \\
\hline
\end{tabular}

Fig. 3. $\bar{C}$.

solution must have that form. For, since we get the lower bound for $\tau^{\prime}(n)$ by simply dividing the total weight of all lines by the "weight" of a single small subsquare, we do not take into account that some of the lines will meet more than one subsquare (i.e., those lines satisfying $g x>1$ ). Thus, if the dual solution is nevertheless "good"-as shown by the primal solution $x$, having almost the same objective function value-the lines meeting more than one subsquare must be precisley those lines having weight almost zero. And these are the lines at the boundary and the two diagonals of $S$. Thus, if we look for integer solutions of $P$, which are "good," we should try to find one that has all nonzero entries nearby the boundary or the diagonals of $S$. In fact, this helped us much in exhibiting the line-covering described below, which is of size $\frac{4}{3} n+O(1)$.

The construction will be described for $n=6 k+1, k \in \mathbb{N}$. We label the rows and columns of $S$ by $i=0, \ldots, 6 k$ and $j=0, \ldots, 6 k$, respectively. Then the $0-1$ incidence matrix $x=\left(x_{i j}\right)$ as introduced previously in this section can be described as follows (see Fig. 4):

$$
\begin{aligned}
& x_{0 j}=1 \Leftrightarrow j=0 \text { or } j=6 k \text { or } j \equiv 2 \bmod 6, \\
& x_{6, j}=1 \Leftrightarrow j=0 \text { or } j=6 k \text { or } j \equiv 4 \bmod 6, \\
& x_{i, 0}=1 \Leftrightarrow i=0 \text { or } i=6 k \text { or } i \equiv 4 \bmod 6, \\
& x_{i, 6 k}=1 \Leftrightarrow i=0 \text { or } i=6 k \text { or } i \equiv 2 \bmod 6 .
\end{aligned}
$$

The elements on the diagonal $x_{6 k-i, i}(i=1, \ldots, 6 k-1)$ are defined as follows:

$$
x_{6 k-i, i}=1 \Leftrightarrow i=1 \text { or } i \equiv 3 \bmod 6 .
$$

In addition to the 1-entries described above, there are some near the second diagonal. These can be described as follows. We let

$$
x_{i, i+2}=1 \Leftrightarrow i=0 \bmod 6
$$

and

$$
x_{i, i+5}=1 \Leftrightarrow i \equiv 1 \bmod 6 .
$$




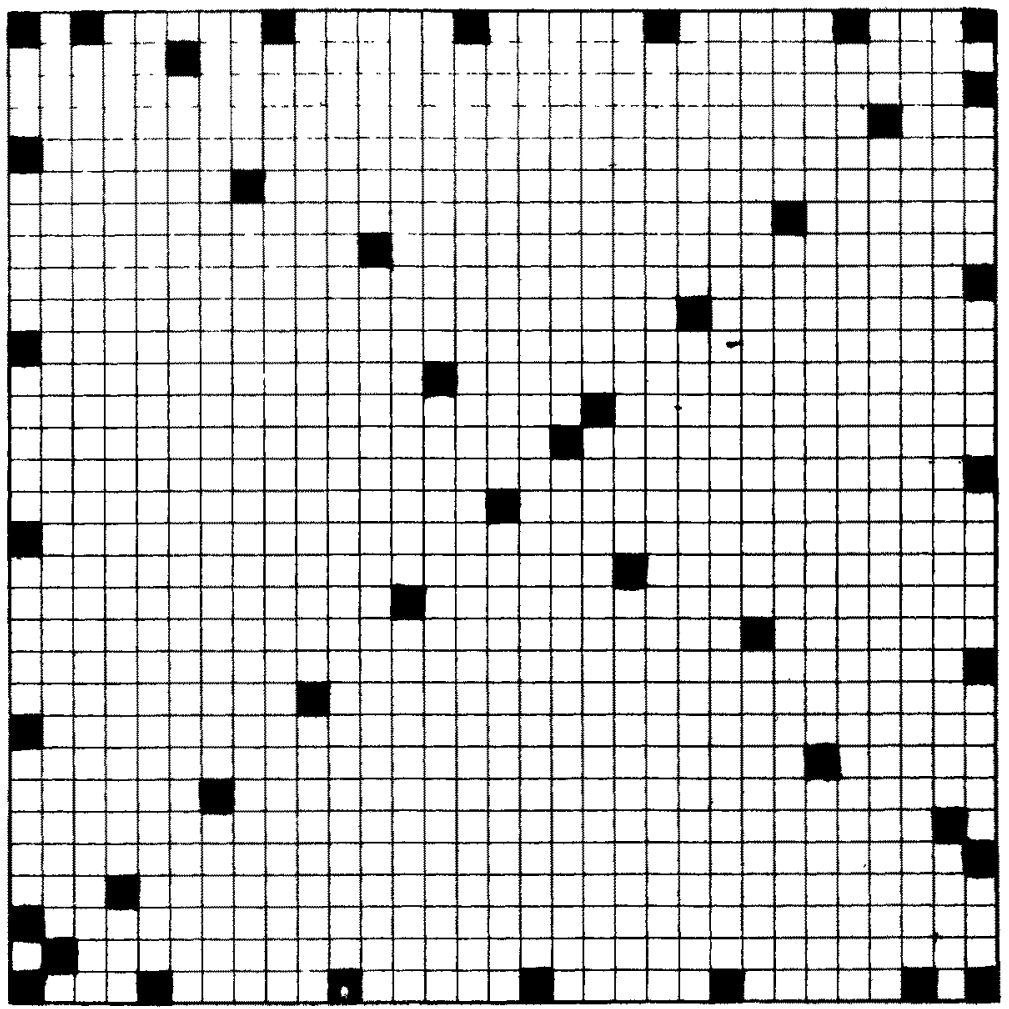

Fig. 4.

It is then straightforward to check that $x$ "blocks" all lines intersecting $S$ which are horizontal, vertical, or parallel to one of the diagonals of $S$. The total number of 1 -entries in $x$ is $\frac{4}{3} n+O(1)$. In case $n \neq 1$ mod 6 one has to add some additional small subsquares at the "corners" of $S$.

\section{Remarks}

1. We spent some time trying to close the gap between the dual solution (equal to $\frac{4}{3} n-\frac{1}{3}$ ) and the primal one, as shown in Fig. 3 (equal to $\frac{4}{3} n+\frac{4}{3}$ ), but we did not succeed. The best we arrived at were some (noninteger) primal solutions of size $\frac{4}{3} n+\frac{2}{3}+O(1 / n)$ for special values of $n\left(n=2^{k}+1\right)$. To describe this construction let us start with the depicted case of $C_{k}, k=2$ (see Fig. 5). Getting the next instance $C_{k+1}$ from $C_{k}$ recursively, the line near the boundary of $C_{k+1}$ (denoted by $\left.L_{k+1}\right)$ is formed as follows: $L_{k+1}$ is the line $L_{k}$ joined together with the reflection of $L_{k}$ with the two ones in the middle replaced by one entry of size $\left(2^{k}-1\right) /\left(2^{k+1}\right)$. From $L_{k+1}$ the size of the remaining nonzero entries on the diagonals are then uniquely determined, taking into account that the weights, added to every nonboundary vertical or horizontal line, are exactly one. 


\begin{tabular}{|r|r|r|r|r|}
\hline 1 & & $1 / 4$ & & 1 \\
\hline & $1 / 2$ & & $1 / 2$ & \\
\hline $1 / 4$ & & $1 / 2$ & & $1 / 4$ \\
\hline & $1 / 2$ & & $1 / 2$ & \\
\hline 1 & & $1 / 4$ & & 1 \\
\hline
\end{tabular}

Fig. 5. $C_{2}$.

\begin{tabular}{|c|c|c|c|c|c|c|}
\hline 1 & $1 / 4$ & $1 / 4$ & $1 / 4$ & $1 / 4$ & $1 / 4$ & 1 \\
\hline $1 / 4$ & $1 / 4$ & & & & $1 / 4$ & $1 / 4$ \\
\hline $1 / 4$ & & $1 / 4$ & & $1 / 4$ & & $1 / 4$ \\
\hline $1 / 4$ & & & $1 / 2$ & & & $1 / 4$ \\
\hline $1 / 4$ & & $1 / 4$ & & $1 / 4$ & & $1 / 4$ \\
\hline $1 / 4$ & $1 / 4$ & & & & $1 / 4$ & $1 / 4$ \\
\hline 1 & $1 / 4$ & $1 / 4$ & $1 / 4$ & $1 / 4$ & $1 / 4$ & 1 \\
\hline
\end{tabular}

Fig. 6.

2. Computing $\tau(n)$ seems to be more difficult than computing $\tau^{\prime}(n)$. Bárány and Füredi [1] report that they have tried to assign weights to a larger set of lines (eight directions instead of four), yielding a lower bound of $1.43 n$ for $\tau(n)$. A primal solution of value $\frac{3}{2} n+1$ is indicated in Fig. 6 . So $\frac{3}{2} n+O(1)$ seems to be the correct value at least for the rational covering problem. In spite of the striking similarity of this solution to the one given in Fig. 3, this time we could not find any integer solution of that size. So it may well be true that Fejes Tóth is right.

\section{References}

1. 1. Bárány and Z. Füredi, Covering all secants of a square, Proceedings of the Colloquia Mathematica Societatis János Bolyai, Siofok, Hungary, 1985 (in honor of L. Fejes Tóth), to appear.

2. L. Fejes Tóth, Remarks on a dual of Tarski's plank problem, Mat. Lapok 25 (1974), 13-20.

3. W. O. Moser and J. Pach, Research Problems in Discrete Geometry, Problem 84, Montréal 1985, mimeographed. 